\section{Genetic Response for Reaction to Verticillium Wilt in Strawberry with Two-stage Family and Genotypic Selection}

\author{
Douglas V. Shaw ${ }^{1}$ and Thomas R. Gordon ${ }^{2}$ \\ University of California, Davis, CA 95616
}

Additional index words. general combining ability, breeding value, test cross, Fragaria xananassa, Verticillium dahliae

\begin{abstract}
Strawberry (Fragaria $\times$ ananassa Duch.) genotypes retained for resistance to Verticillium wilt (Verticillium dahliae Kleb.) after two cycles of a two-stage (TS) selection procedure consisting of full-sib family selection followed by within-family selection of individuals, and genotypes retained for resistance using genotypic mass (GM) selection were crossed to a common set of moderately susceptible genotypes. The relative resistance of the seedlings from these progenies was compared using a resistance score and the percentage of stunted plants. Although the two sets of resistant parents had performed similarly in genotypic comparisons, those genotypes selected using the TS procedure yielded test cross offspring with significantly higher resistance scores $(\bar{X}=3.84 \pm 0.09$ vs. $\bar{X}=3.46 \pm 0.09$, $t=3.11 * *)$ and significantly lower rates of plant stunting $(\bar{X}=38.1 \% \pm 3.1$ vs. $\bar{X}=\mathbf{5 0 . 2 \%}$ $\pm 2.9, t=2.87 * *)$ than the parents chosen using GM selection. Further resolution using analysis of variance and general combining ability (GCA) estimates showed that these between-set differences resulted from higher resistance breeding values for parents selected using the TS procedure. The five genotypes with largest GCA for resistance score and four of the five genotypes with minimum GCA for percentage stunting were obtained by TS selection.
\end{abstract}

Plant stunting or mortality resulting from Verticillium wilt (Verticillium dahliae Kleb.) infection of strawberry (Fragaria $\times$ ananassa Duch.) can be a major obstacle to commercial fruit production in California (Thomas, 1932; Wilhelm and Koch, 1956). Successful management of Verticillium has been achieved through soil fumigation of fruit production fields (Wilhelm and Koch, 1956; Wilhelm et al., 1974; Paulus, 1990) together with annual planting (Wilhelm and Paulus, 1980), avoidance of heavily infested soils (Strand, 1994), and careful control of disease progression in runner plant propagation nurseries (Gordon et al., 2002). Although most progress in controlling Verticillium during the last 50 years has been made through horticultural manipulations, breeding for resistance may be an increasingly important component of the management strategy for this disease if regulations further restrict the availability and use of effective soil fumigants (Watson et al., 1992).

Efforts to breed for Verticillium wilt resistance in California trace to early in the program's history (Bringhurst et al., 1961; 1968). More recently, Shaw et al. (1996) demonstrated substantial genetic variation

Received for publication 22 Jan. 2002. Accepted for publication 14 May 2002. We thank Lassen Canyon Nursery for their generous assistance in conducting the nursery phases of this research.

${ }^{1}$ Pomology Dept., Univ. of California, Davis, CA 95616.

${ }^{2}$ Plant Pathology Dept., Univ. of California, Davis, CA 95616 . for resistance to Verticillium wilt within the Univ. of California's (UC) breeding population, but cautioned that little resistance had been incorporated into commercial cultivars preferred by the industry to date. These recent genetic analyses also demonstrated that dominance effects were important, perhaps responsible for half of the genetic variance for resistance detected. The presence of substantial dominance genetic variation suggested an opportunity for are frequently more efficient than genotypic selection for improving breeding value when dominance components of variance are large (Falconer and MacKay, 1996).

Further evaluations of first-generation selection results (Shaw et al., 1997) demonstrated substantial response to selection among full-sib families for two resistance traits. Due to the presence of substantial within-family genetic variance for Verticillium resistance, a twostage selection procedure was recommended, in which individuals from superior progenies are further tested using replicated plots of inoculated runner plants. Other benefits of this two-stage procedure include the logistical advantage of dealing only with noninfected stock in propagation nurseries (Shaw et al., 1997), and opportunities for efficiently combining selection for resistance with the evaluation of seedlings for horticultural traits.

An alternative strategy to multiple stage selection is genotypic mass selection, using direct clonal tests for resistance without prior family selection. Such genotypic evaluations are facilitated in strawberry by easy vegetaimproved selection efficiency using an emphasis on family performance. Such methods tive propagation through runner plants. Direct genotypic evaluation is often less efficient for population improvement than methods that incorporate family testing (Shaw, 1996), but offer logistical advantages. In particular, resistance screening can be conducted on genotypes with known superior performance for horticultural traits.

Ultimately, the efficient incorporation of genetic resistance into commercially acceptable cultivars will mandate the development of entire populations of genotypes with high levels of resistance that in addition possess acceptable horticultural performance. The objective of the experiment reported here was to compare the parental performance, or breeding value, for resistance to Verticillium wilt of genotypes selected using individual genotypic performance evaluations (genotypic mass selection) with that of genotypes retained over two cycles of full-sib family selection followed by subsequent clonal testing of individuals from superior families (two-stage selection).

\section{Materials and Methods}

Forty-nine bi-parental crosses, or full-sib families, were generated in Jan. 2000 using a factorial mating design (Comstock and Robinson, 1948) between 10 resistant genotypes and five moderately susceptible genotypes. The 10 resistant parents represented two sets of five genotypes, with each set chosen using differing selection methods. Several other features, including selection intensities, differed among the resistant sets generated by the two selection methods, and direct comparison of the relative effectiveness of each method for obtaining genotypic resistance was not a primary goal of this experiment. Rather, the ultimate goal of this study was the comparison of parental potential, or resistance breeding value, for sets of genotypes with similar levels of genotypic performance. Genotypic resistance scores for all sets of parents are given in Table 1 and their origin is described further below.

One set of resistant parents was chosen after two cycles of two-stage (TS) selection (i.e., the seedling population in the second cycle was generated by crosses among the resistant selections retained in the first). In the first stage of each selection cycle, the best full-sib families were chosen based on their mean resistance scores (Shaw et al., 1997); in the second stage runner plants from seedlings chosen at random with respect to Verticillium resistance from these superior progenies were inoculated and individual genotypes retained based on their individual, or genotypic, resistance scores. Full-sib families were evaluated in both TS selection cycles using two plots of 10 seedlings each, and evaluations were performed after inoculation with $10-15$ mL of a condial suspension containing $\approx 10^{6}$ conidia/mL of 3-5 isolates of V. dahliae (Shaw et al., 1996, 1997). Family selection intensities $\left(i_{f}\right)$ were 1.7 and 0.37 for the two cycles, respectively. Family selection in the second cycle was conducted by elimination of only the most susceptible crosses, as the overall resistance in this population was high. Individual genotypes retained from the 
Table 1. Genotypic Verticillium resistance scores and estimates of the percentage of severely stunted plants $( \pm \mathrm{SE})$ for two sets of resistant parents and one set of susceptible parents used in test crosses to evaluate two-stage and genotypic mass selection methods.

\begin{tabular}{lcc}
\hline Parent selection criterion & Resistance score & Stunted (\%) \\
\hline Two-stage selection & $4.47 \pm 0.15$ & $28.5 \pm 7.4$ \\
Genotypic mass selection & $4.34 \pm 0.19$ & $37.8 \pm 8.4$ \\
Susceptible control & $2.33 \pm 0.24$ & $89.7 \pm 4.7$ \\
\hline
\end{tabular}

selected full-sib families were assessed using two plots of five runner plants, respectively, following immersion for $10 \mathrm{~m}$ in an aqueous suspension containing $\approx 10^{6}$ conidia $/ \mathrm{mL}$ of $3-5$ isolates of $V$. dahliae (Shaw et al., 1996), and within-family selection intensities $\left(i_{\mathrm{wf}}\right)$ were $\approx 1.2$ and 1.3 for the two cycles, respectively. The seedlings retained initially from superior full-sib families were selected for fruit quality traits prior to their genotypic assessments, but this is not expected to affect their performance for resistance characteristics.

A second set of five resistant parents was generated using genotypic mass (GM) selection (Shaw et al., 1996). Parents for this set were obtained by screening 172 genotypes representative of cultivars and advanced selections either in use as parents or under consideration for release as cultivars by the UC strawberry improvement program between 1997 and 2001. Each genotype was tested using two plots of five runner plants, using procedures identical to the second stage of the TS selection procedure described above. All resistant selections in this set were tested in at least two seasons. The genotypes used as parents from this population were chosen to control relatedness as well as for superior resistance, and reflect a selection intensity $(i)$ of 2.2.

Five moderately susceptible genotypes were chosen from the cultivars and advanced selections used as a base population for GM selection and used in test crosses to evaluate the breeding value of the resistant selections. These genotypes represent essentially a random sample of the more susceptible $50 \%$ of the 172 genotypes from which GM selection selections were obtained.

Seedlings from each bi-parental cross were established in experimental plots located at the Wolfskill Experimental Orchard near Davis, Calif., on 26 Sept. 2000 as described previously (Shaw et al., 1997). Briefly, soil was prepared for planting and treated with 2 methyl bromide : 1 trichloronitromethane (chloropicrin) at 392 $\mathrm{kg} \cdot \mathrm{ha}^{-1}$ in Aug. 2000. Immediately prior to transplanting in the field, 20 seedlings from each progeny were inoculated with $10 \mathrm{~mL}$ of a conidial suspension containing $10^{6}$ conidia $/ \mathrm{mL}$ of three isolates of $V$. dahliae obtained from symptomatic strawberry plants near Watsonville, Calif. Seedlings were transplanted to the field, in a randomized complete-block design with a single plot of 10 seedlings in each of two blocks, then evaluated for disease symptoms in Spring 2001 as previously described and reported (Shaw et al., 1996).

Disease symptoms were scored as the number of dead or seriously stunted plants per 10-plant plot, and on the basis of a subjective phenotypic resistance score assigned of susceptible parents.

Significant at $P<0.01$. to each plot. The subjective resistance score was assigned on a scale from 1 to 5 , where $1=$ severe Verticillium wilt symptoms (in most cases, complete mortality) and $5=$ no detectible disease symptoms. Evaluations were performed on five dates during the spring after planting: 3 Apr., 26 Apr., 17 May, 1 June, and 15 June 2001. Further statistical analyses were conducted using the average values for each variable over the five observation dates. The numbers of stunted, dead, or both plants were converted to percentages prior to analysis.

Initial comparisons were made between the two sets of full-sib families generated with parents from differing selection methods using Student's $t$ test. Differences among the sets of parents were further resolved by factorial analysis of variance (ANOVAs) with selection method, general combining ability (GCA) for resistant and susceptible parents within selection method, and specific combining ability (SCA) treated as fixed effects and tested using SAS procedure GLM and Type III sums of squares (SAS Institute, 1988). The ANOVAs were followed by estimation and comparison of GCA values for resistant parents using the GAREML software program (Huber, 1993).

\section{Results and Discussion}

Genotypes retained after TS selection had slightly higher resistance scores and lower stunting percentages in runner plant inoculation trials than those obtained by GM selection for both traits (Table 1), but these set means differed by less than one standard error (SE), and differences were not statistically significant. The two selection procedures thus provided sets of parents with similar genotypic performance for resistance. Conversely, genotypes chosen using the TS selection produced test cross offspring with highly significant $(P<0.01)$ increases in resistance score $(\bar{X}=3.84 \pm 0.09$ vs. $\bar{X}=3.46 \pm 0.09)$ and decreases in percentage of severe stunting/mortality $(\bar{X}=38.1 \pm 3.1$ vs. $\bar{X}=50.2 \pm 2.9)$ compared with offspring from parents obtained using GM selection (Table 2). Although both methods of selection were successful in identifying individuals with outstanding genotypic resistance, TS selection was superior in developing resistant parents. Together, the similarity of parental sets for genotypic resistance traits and the significant differences between their resistance breeding values suggest that the two selection methods capture very different fractions of the genetic variation initially available in this population (Shaw et al., 1996).

Further resolution by ANOVA also demonstrated highly significant differences for both resistance traits between the offspring of parents obtained with the two selection methods (Table 3); no within-set differences were found among the resistant parents for GCA . These results imply that each of the selection methods produced a set of genotypes with very homogeneous breeding value for resistance and that essentially all of the differences among resistant genotypes for parental potential were due to the selection method used for their generation. Highly significant differences were detected for GCA among the susceptible parents, demonstrating substantial variation for resistance among these genotypes. Similarly, SCA within selection method was significant $(P<0.05)$ for the percentage of stunted plants, demonstrating that some of the genetic differences within each set were due to nonadditive genetic effects.

Comparisons of individual GCA estimates from a combined factorial analysis of all 10 resistant parents further illustrate the differences between the genotypic sets for resistance breeding value. The five genotypes with largest GCA for resistance score and four of the five genotypes with minimum GCA for percentage

Table 2. Verticillium resistance scores and estimates of the percentage of severely stunted plants $( \pm \mathrm{SE})$ for seedlings from two resistant sets of parents crossed to a common set

\begin{tabular}{lccc}
\hline Parent selection criterion & $\mathrm{N}$ & Resistance score & Stunted (\%) \\
\hline Two-stage selection & 48 & $3.84 \pm 0.09$ & $38.1 \pm 3.1$ \\
Genotypic mass selection & 50 & $3.46 \pm 0.09$ & $50.2 \pm 2.9$ \\
Student's $t$ test & & $t=3.11^{* *}$ & $t=2.87^{* *}$ \\
\hline${ }^{* *}$ Significant at $P<0.01$ & & &
\end{tabular}

Table 3. Mean squares from the analysis of variance of two variables that describe resistance to Verticillium dahliae Kleb. for seedlings from two sets of resistant parent genotypes crossed to a common set of susceptible testers.

\begin{tabular}{|c|c|c|c|}
\hline \multirow[b]{2}{*}{ Source } & \multirow[b]{2}{*}{ df } & \multicolumn{2}{|c|}{ Disease symptom variable } \\
\hline & & Resistance score & Stunted (\%) \\
\hline$\overline{\text { Block }}$ & 1 & $1.10^{*}$ & $1800.0^{* *}$ \\
\hline Selection method (S) & 1 & $3.69^{* *}$ & $3663.3^{* *}$ \\
\hline Resistance parent GCA/S & 8 & 0.26 & 487.3 \\
\hline Susceptible parent GCA/S & 8 & $1.40^{* *}$ & $1338.8^{* *}$ \\
\hline $\mathrm{SCA} / \mathrm{S}$ & 31 & 0.36 & $423.2^{*}$ \\
\hline Within family & 48 & 0.22 & 247.9 \\
\hline
\end{tabular}

*,** Significant at $P<0.05$ and 0.01 , respectively. 
Table 4. GCA estimates for Verticillium resistance scores and the percentage of severely stunted plants for sets of resistant parents obtained with two-stage and genotypic mass selection methods.

\begin{tabular}{llcc}
\hline & & \multicolumn{2}{c}{ GCA estimate } \\
\cline { 3 - 4 } Parent selection method & Genotype & Resistance score & Stunted (\%) \\
\hline Two-stage selection & CA250201 & 0.10 & -7.2 \\
& CA255203 & 0.14 & -8.8 \\
& CA259201 & 0.09 & 5.0 \\
& CA263203 & 0.05 & -5.1 \\
Genotypic mass selection & CA267202 & -0.09 & -3.2 \\
& CA1001 & -0.10 & 5.0 \\
& CA3011 & -0.15 & 1.2 \\
& CA116005 & -0.29 & 3.1 \\
& CA145002 & 0.05 & 8.7 \\
& CA257607 & & 1.2 \\
\hline
\end{tabular}

stunting were obtained by TS selection (Table 4). Furthermore, significant correlations were not detected between the original genotypic value obtained by testing runner plants and individual GCA estimates for resistance score $(r=-0.16, P=0.66)$ or percentage stunting $(r=-0.16 . P=0.67)$ within the combined resistant parents. These results confirm that the significant differences detected among offspring from differing parental sets noted above were due to differences in resistance breeding value, and support the conclusion that the selection methods compared here have exploited different fractions of the genetic variation initially available.

It is important to reiterate that both selection methods produced genotypes with high resistance potential compared with their corresponding unselected populations. General combining ability estimates for resistance traits in this trial reflect differences in parental potential among the more Verticillium-resistant genotypes available. Regardless, our results suggest that individual performance in genetic resistance to Verticillium may only partially predict performance for breeding value and argue for use of methods that incorporate either family selection or progeny testing, at least when the objective is rapid development of entire populations of resistant individuals.

\section{Literature Cited}

Bringhurst, R.S., P.E. Hansche, and V. Voth. 1968. Inheritance of Verticillium wilt resistance and the correlation of resistance with performance traits. Proc. Amer. Soc. Hort. Sci. 92:369-375.

Bringhurst, R.S., S. Wilhelm, and V. Voth. 1961. Pathogen variability and breeding Verticillium wilt resistant strawberries. Phytopathology 51: 786-794.

Comstock, R.E. and H.F. Robinson. 1948. The components of genetic variation in populations of bi-parental progenies and their use in estimating the average degree of dominance. Biometrics 4:254-266.

Falconer, D.S. and T. MacKay. 1996. Introduction to quantitative genetics. Chapters 13 and 17 .

Gordon, T.R., S.C. Kirkpartick, D.V. Shaw, and K.D. Larson. 2002. Differential infection of mother and runner plant generations by Verticillium dahliae in a high elevation strawberry nursery. HortScience 37:927-931.

Huber, D.C. 1993. Optimum mating design and Longman Press. New York. optimum techniques for analysis of quantitative traits in forest genetics. PhD Diss., Univ. of Florida, Gainesville.

Paulus, A.L. 1990. Fungal diseases of strawberry. HortScience 25:885-889.

SAS Institute, Inc. 1988. SAS/STAT users guide, release 6.03 ed. SAS Inst., Cary, N.C.

Shaw, D.V. 1996. Use of quantitative genetics for strawberry cultivar development. In: Marvin P. Pritts, Craig K. Chandler, and Timothy E. Crocker (eds.). Fourth North Amer. Strawberry Conf., Orlando, Fla., 15-17 Feb. 1995. Univ. of Florida Press.

Shaw, D.V., W.D. Gubler, K.D. Larson, and J.Hansen. 1996. Genetic variation for field resistance to Verticillium dahliae evaluated using genotypes and segregating progenies of California strawberries. J. Amer. Soc. Hort. Sci. 121:625-628.

Shaw, D.V., W.D. Gubler, J. Hansen, and K.D. Larson. 1997. Response to family selection for field resistance to Verticillium dahliae in California strawberries. J. Amer. Soc. Hort. Sci. 32:711-713.

Strand, L.L. 1994. Integrated pest management for strawberries. Univ. of California, DANR Publ. 3351. Univ. of Calif. Press, Oakland.

Thomas, H.E. 1932. Verticillium wilt of strawberries. Univ. Calif. Agr. Exp. Sta. Bul. 530.

Watson, R.T.,D.L.Albritton, S.O. Anderson, and S.E. Bapty. 1992. Methyl bromide: Its atmospheric science, technology, and economics. Montreal Protocol Assessment Summary, United Nations Environ. Program, Nairobi, Kenya.

Welch, N.C. 1989. Strawberry production in California. Coop. Ext. Lftt. 2959, Univ. of California, Oakland.

Wilhelm, S. and E.C. Koch. 1956. Verticillium wilt controlled. Calif. Agr. 10:3-14.

Wilhelm, S. and A.O. Paulus. 1980. How soil fumigation benefits the California strawberry industry. Plant Dis. 64:264-270.

Wilhelm, S., R.C. Storkan, and J.M. Wilhelm. 1974. Preplant soil fumigation with methyl bromide-chloropicrin mixtures for control of soil-borne diseases of strawberries-A summary of fifteen years of development. Agr. and Environ. 1:227-236. 